\title{
Comparison of ozone and lidocaine injection efficacy vs dry needling in myofascial pain syndrome patients
}

This article was published in the following Dove Press journal: Journal of Pain Research

\author{
Seyed Ahmad Raeissadat' \\ Seyed Mansoor Rayegani ${ }^{2}$ \\ Fatemeh Sadeghi $^{3}$ \\ Shahram Rahimi-Dehgolan ${ }^{3}$ \\ 'Clinical Development Research \\ Center of Shahid Modarres Hospital, \\ Physical Medicine and Rehabilitation \\ Research Center, Shahid Beheshti \\ University of Medical Sciences, \\ Tehran, Iran; ${ }^{2}$ Physical Medicine and \\ Rehabilitation Research Center, \\ Shohada-e-Tajrish Hospital, Shahid \\ Beheshti University of Medical \\ Sciences, Tehran, Iran; ${ }^{3}$ Physical \\ Medicine and Rehabilitation Research \\ Center, Shahid Beheshti University of \\ Medical Sciences, Tehran, Iran
}

Purpose: Myofascial pain syndrome (MPS) is a common musculoskeletal disorder among young adults associated with presence of myofascial trigger points. We aimed to evaluate efficacy of ozone injection (OI) in MPS patients, compared with two currently used methods including lidocaine injection (LI) and dry needling (DN).

Patients and methods: In this single-blinded study, a total of 72 eligible patients were included and then randomly divided into three equal groups: DN, OI, and LI. All patients received treatment in three weekly sessions. Visual analog scale (VAS) for pain, cervical lateral flexion, pain pressure threshold (PPT), and neck disability index (NDI) were the main outcome measures, which were evaluated at baseline and at 4 weeks after injections. Analytic results were demonstrated as both within- and between-groups mean difference (MD).

Results: Sixty two patients finished the study, 20 participants in both the DN and LI groups, and 22 persons in OI group. Distribution of all demographics and baseline clinical variables were relatively similar among groups. All three interventions were remarkably effective in improving patients' pain and PPT. Significant decrease in VAS (MD=-3.6 \pm 1.4$)$ and increase in PPT (MD=7.2 \pm 5.1$)$ within 4 weeks follow-up confirmed this finding. Also, NDI had similar significant improvement $(\mathrm{MD}=-9.9 \pm 8.7)$, but lateral flexion range did not show remarkable increase. There was also a statistically significant difference among three methods' efficacy on VAS, NDI, and PPT, favoring OI and LI.

Conclusion: In summary, this data showed that in short-term follow-up, all three methods were significantly effective in MPS treatment; however, OI and LI groups had slightly better results than the DN group, with no remarkable preference between them.

Keywords: myofascial trigger points, intra-muscular ozone injection, wet needling

\section{Introduction}

Myofascial pain syndrome (MPS), as a common type of repetitive strain injury, is an important condition that presents as fatigue and musculoskeletal pain in multiple tender/trigger points. ${ }^{1-3}$ MPS is associated with the presence of some myofascial trigger points (MTrP) that are found in a taut band inside the muscles; sometimes MTrP has a local twitch response following a rapid snapping stimulus or inserting a needle. ${ }^{1,4-6}$ The criteria for diagnosis and the clinical importance have evolved over time; by applying Simons' 7-point clinical criteria, the diagnosis of MPS has relied mainly on the clinical history and a careful physical examination by a trained clinician. ${ }^{7}$ Despite these features, diagnosis is still unclear and confusing. The prevalence is unclear due to lack of well-defined diagnostic criteria. In some studies, it has been seen in about
Correspondence: Shahram

Rahimi-Dehgolan

No. 1998734383, Physical Medicine and Rehabilitation, Shahid Modarres Hospital, Kaj Square, Saadat Abad Street, Tehran, Iran

Tel +98 2l 22074090

Email shahram.rahimi.dehgolan@gmail. com 
$30 \%-46 \%$ of patients at pain clinics or $15 \%$ of those presenting to general medical clinics, particularly among young- or middle-aged populations. ${ }^{8,9}$

MPS has been treated using various methods, ranging from exercise and oral analgesics to various physical modalities including transcutaneous electrical stimulation, infrared, ultrasound, manual pressure and massage, acupuncture, anesthetic injections, and even dry needling (DN) accompanied with no drug injection. ${ }^{9} \mathrm{DN}$ is a procedure in which an acupuncture-like needle is inserted into the muscle to possibly frustrate an MTrP. DN might modulate regional levels of important chemicals including bradykinin, calcitonin gene-related peptide, and substance P. However, the precise mechanism responsible for its efficacy is still unclear. Local lidocaine injection (LI) also can induce a similar analgesic pathway as well as the contribution of DN effects. A metaanalysis study, comparing the effectiveness of DN to control methods, revealed that LI might be superior to DN alone, for both immediate pain relief and at 4 weeks after treatment. ${ }^{6}$

Despite this wide range of therapeutic methods, strong evidence to guide treatment methodologies such as use of local anesthetics, saline, or injection of other products, is lacking. ${ }^{9-14}$ Recently, there has been increasing evidence supporting the role of ozone injection (OI) in the management of some musculoskeletal disorders like lumbosacral disc herniation, knee osteoarthritis and meniscal injury, shoulder disorders, and so on. This gas is now available in a solution of oxygen/ozone. As multiple studies has proven, ozone can improve tissue oxygenation, inhibit inflammatory mediators by downregulation of tumor necrosis factor (TNF) and TNFR2, and induce a moderate analgesic effect through phosphodiesteraseA2 blockage. ${ }^{15-24}$ On the other hand, some theories explaining energy expenditure and muscle fatigue in MTrPs have been introduced like Simons' Integrated Trigger Point Hypothesis on sensitizing neuroreactive substances and Cinderella Hypothesis, ${ }^{7}$ which imply that using ozone in low concentration, as an activator of the enzymatic scavenger system (catalase, glutathione-peroxidase, and superoxide dismutase), can break down oxygen-free radicals and probably could be beneficial in MPS treatment. ${ }^{7}$ Thus, in this randomized controlled trial (RCT), we aimed to evaluate ozone efficacy and safety in MPS patients, compared to two methods currently used: DN and LI. ${ }^{7,25}$

\section{Patients and methods}

This single-blind RCT was conducted with three parallel groups in the Physical Medicine and Rehabilitation clinic of Shahid Modarres hospital in Tehran from March
2016 to August 2016. We had registered this trial in the Iranian Registry of Clinical Trials with ID number: IRCT2016042113442N10. Informed written consent was obtained from the subjects who met the inclusion criteria. This study was also approved by the ethics committee of Shahid Beheshti University of Medical Sciences, Tehran, Iran (No IR.SBMU.SM.REC.1394.168).

\section{Inclusion criteria}

All patients presenting with chronic nonspecific neck pain were evaluated by an expert physiatrist with 15 years of experience in the field of clinical musculoskeletal disorders; eventually, subjects with clinically confirmed diagnosis of MPS (based on Simons' criteria) who had at least one trigger point in their upper trapezius muscle were enrolled. Of them, 72 consecutive patients were included according to our inclusion criteria, which were as follows:

1. Age $25-60$ years

2. Symptoms lasting $>3$ months despite conservative treatments

3. Presence of active trigger points in the upper trapezius muscles

4. Presence of at least one taut band on manual palpation

5. Triggering of the pain using finger point pressure

\section{Exclusion criteria}

The exclusion criteria were as follows: presence of any history of cervical radiculopathy or degenerative condition, any surgery or trauma to the neck during last year, previous injection for MPS treatment during last 6 months, confirmed diagnosis of cognitive disorders, fibromyalgia, rheumatoid arthritis, and/or metabolic diseases like hypothyroidism and diabetes mellitus.

\section{Interventions}

Seventy two eligible participants were randomly assigned to three equal groups using block randomization method: OI, wet needling by LI, and DN alone. Participants were all blinded to the concealed allocation of methods. Concealment was performed using some sealed paper envelopes. In all patients, trigger points were identified by manual palpation, and in those with two or more trigger points, the most painful one was chosen. MTrP site was marked and the location was also recorded regarding to its distance from anatomic landmarks in possible cases where the marker had washed off. Patients were lying in prone position; the injection site was sterilized, and the point was grabbed between the thumb 
and index fingers. For all patients, three consecutive weekly rounds of treatment were performed, using a 22 gauge, 1.25 inch needle, by an expert physiatrist.

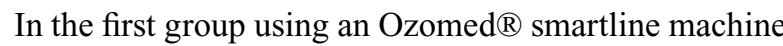
(manufactured by Kastner, Praxisbedarf GmbH, Medizintechnik. Germany), 8 cc of oxygen/ozone gas was injected in the affected MTrP with a concentration of $15 \mu \mathrm{g} / \mathrm{mL}$. The second group (LI) received $2 \mathrm{cc}$ of lidocaine $2 \%$. In the DN group, the needle was inserted into the MTrP, then was withdrawn to reach the subcutaneous tissue, and then was similarly inserted and withdrawn in different directions to frustrate the point. Regardless of the method used, all patients were advised to perform some modification in their daily activities to have lasting relief like avoiding muscle overuse in cervical and postural muscles during the performance of lowintensity activities of daily living and sedentary work, and they were instructed to do an exercise program consisting of trapezius stretching techniques and muscle relaxation training. Three groups were also advised to use oral medication (only acetaminophen tablets with maximum dose of $4 \mathrm{~g} / \mathrm{d}$ ) and ice massage, if it was needed up to the next 48 hours after injection. Additionally, one of the authors (Shahram Rahimi Dehgolan) was responsible for weekly overseeing of exercise and lifestyle modification performance via phone call.

\section{Outcome measurements}

Data on demographic characteristics of the subjects such as age, gender, body mass index (BMI), and duration of symptoms were recorded. To assess the patient's response, four clinical parameters were measured twice, once before the treatment and the second time 4 weeks after the last injection.

1. Severity of the pain evaluated by the visual analog scale (VAS), rated from 0 (no pain) to 10 (the worst pain).

2. Irritability of the trigger point according to the Pain Pressure Threshold (PPT) defined as the minimum amount of compression on the point that reproduces the pain. To measure the PPT, an Algometer device was placed perpendicularly on the location of the trigger point and the pressure was increased by $1 \mathrm{~kg} / \mathrm{cm}^{2} / \mathrm{s}$ until the patient feels the pain. The measurements were repeated three times with 30-second intervals and the mean value of the three measurements was recorded.

3. Range of motion (ROM) in neck lateral flexion movement measured according to the maximum angle that the patient could laterally bend his or her neck to the right and left side, using three consecutive times of goniometry and recording maximum value of them. Mean of highest values of both directions was recorded as the final amount.

4. Self-reported disability index of patients according to the neck disability index (NDI). NDI was assessed by the neck pain questionnaire that included 10 questions regarding the severity of neck pain, its impact on sleeping, driving, etc. Each question was scored from 0 to 5 and the total score was measured from 50 and was finally reported in percent (\%). A higher percent of NDI was an indicator of more disability and pain.

\section{Data analysis}

The minimum sample size for each group was calculated as 20 ; then with the assumption of $15 \%-20 \%$ dropout rate, we recruited 24 patients in each group and commenced with total of 72 participants. Descriptive analysis was reported as frequency and percent for qualitative variables and as mean and SD for quantitative variables. Then, effect size was assessed as raw mean difference (MD) within each group and between the groups. Analysis was done using SPSS (SPSS, Inc, Chicago, IL, USA), version 22.0 by means of paired and student $t$-tests, analysis of variance, and $\chi^{2}$, for quantitative and qualitative variables, respectively. $p$-value $<0.05$ was considered as statistically significance.

\section{Results}

A total of 72 patients were included in the study; among them, 10 subjects exited the survey due to different reasons like willingness to continue on physiotherapy and personal problems (unrelated to our intervention). Eventually, 62 patients, 20 participants in both the DN and LI groups, and 22 patients in the ozone group finished the study (Figure 1). The mean age of patients was $39.4 \pm 7.9$ years. Forty-eight patients $(77.4 \%)$ were female and fourteen $(22.6 \%)$ were male. The mean duration of symptoms was calculated as $4.4 \pm 1.2$ months. Distribution of demographic variables like age, weight, BMI, and baseline clinical variables in three groups is demonstrated in Table 1. There was no statistical difference among groups, and they could be considered similar at first.

As demonstrated in Table 2, all three methods were remarkably effective in improving patients' signs and symptoms including pain and pain pressure threshold (PPT). Significant decrease in VAS $(\mathrm{MD}=-3.6 \pm 1.4, p$-value $=0.001)$ and increase in PPT $(\mathrm{MD}=7.2 \pm 5.1, p$-value $=0.001)$ after 4 weeks follow-up confirmed this finding. Also, NDI showed a similar significant improvement $(\mathrm{MD}=-9.9 \pm 8.7$, 


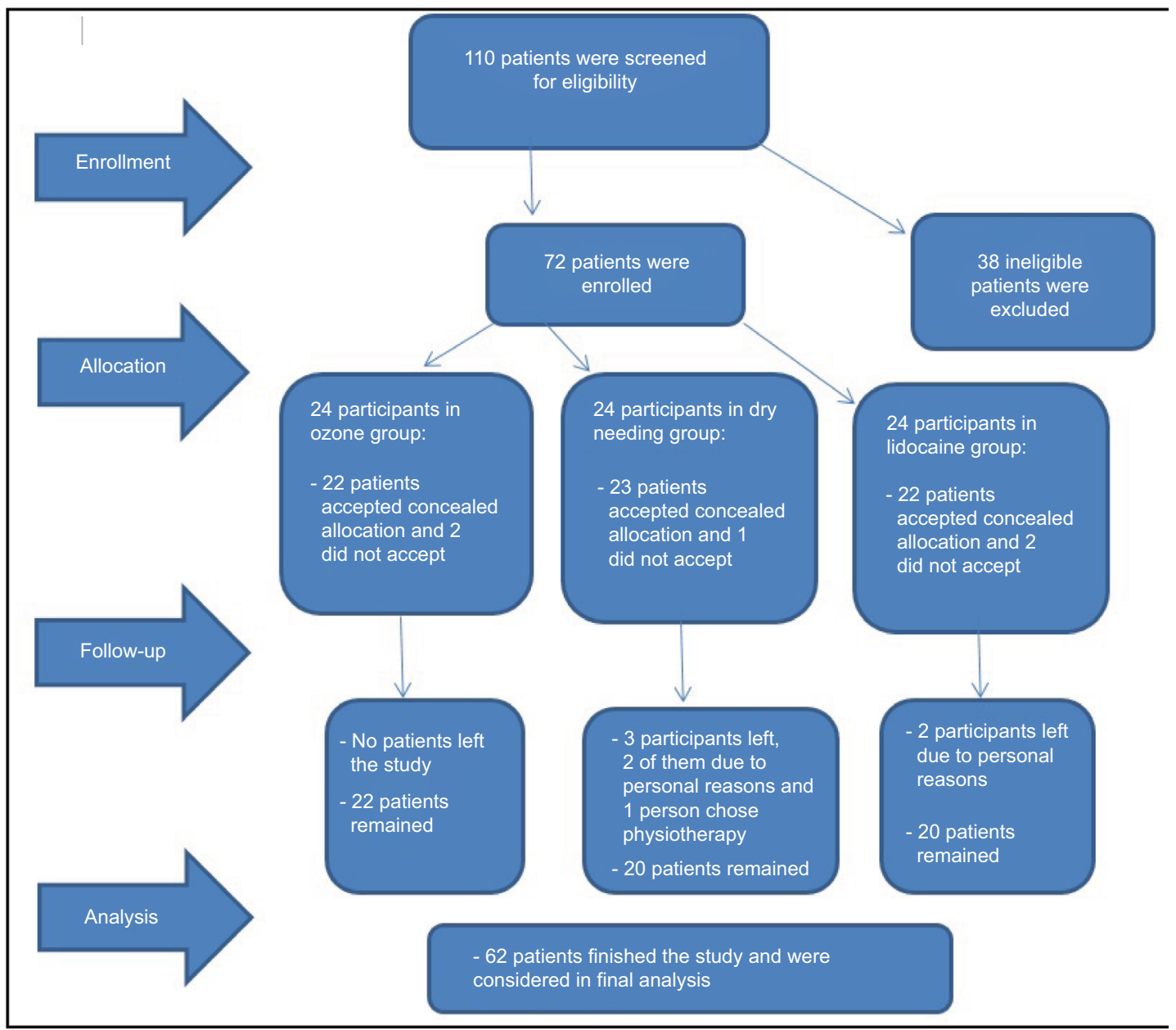

Figure I Flow diagram of the study population.

Table I Demographic distribution and comparison of three groups

\begin{tabular}{|c|c|c|c|c|c|}
\hline & DN & OI & LI & Total & $p$-value \\
\hline Number of cases & 20 & 22 & 20 & 62 & \\
\hline Age (years) & $41.6 \pm 6.8$ & $37.6 \pm 8.8$ & $39.4 \pm 7.7$ & $39.4 \pm 7.9$ & 0.27 \\
\hline Gender & & & & & 0.80 \\
\hline Female & $16(80 \%)$ & $16(72.7 \%)$ & $16(80 \%)$ & 48 (77.4\%) & \\
\hline Male & $4(20 \%)$ & $6(27.3 \%)$ & $4(20 \%)$ & $14(28.6 \%)$ & \\
\hline BMI $\left(\mathrm{kg} / \mathrm{m}^{2}\right)$ & $23.7 \pm 1.4$ & $25.9 \pm 6.0$ & $24.6 \pm 2.5$ & $24.7 \pm 4.0$ & 0.193 \\
\hline Height (cm) & $162.5 \pm 3.7$ & $164.5 \pm 7.9$ & $164.0 \pm 8.0$ & $163.7 \pm 6.8$ & 0.632 \\
\hline Chronicity (month) & $4.6 \pm \mathrm{I} .4$ & $4.4 \pm 1.3$ & $4.4 \pm 1.2$ & $4.4 \pm 1.3$ & 0.78 \\
\hline Affected side (Rt:Lt ratio) & 12:8 (1.5) & | $4: 8(1.75)$ & $12: 8(1.5)$ & $38: 24(1.58)$ & 0.96 \\
\hline VAS & $6.4 \pm 0.7$ & $6.3 \pm 1.2$ & $6.3 \pm 0.9$ & $6.3 \pm 0.9$ & 0.987 \\
\hline PPT $\left(\mathrm{kg} / \mathrm{cm}^{2}\right)$ & $27.8 \pm 3.7$ & $28.7 \pm 7.0$ & $29.7 \pm 5.7$ & $28.7 \pm 5.6$ & 0.592 \\
\hline NDI & $46.3 \pm 9.1$ & $49.6 \pm I I .4$ & $51.1 \pm 7.0$ & $49.0 \pm 9.5$ & 0.273 \\
\hline ROM (degree) & $32.8 \pm 4.7$ & $34.0 \pm 4.2$ & $33.6 \pm 6.4$ & $33.5 \pm 5.1$ & 0.760 \\
\hline
\end{tabular}

Abbreviations: BMI, body mass index; DN, dry needling; LI, lidocaine injection; NDI, neck disability index; OI, ozone injection; PPT, pain pressure threshold; ROM, range of motion; VAS, visual analog scale. 
Table 2 Comparison of efficacy among three groups before and after intervention

\begin{tabular}{|c|c|c|c|c|c|c|c|c|c|}
\hline & \multicolumn{3}{|l|}{ Total } & \multicolumn{2}{|l|}{$p$-value } & \multicolumn{3}{|l|}{ DN } & \multirow[t]{2}{*}{$p$-value } \\
\hline & Before & After & MD & $\begin{array}{l}\text { Paired } t \text {-test } \\
\text { within groups }\end{array}$ & $\begin{array}{l}\text { ANOVA among } \\
\text { three groups }\end{array}$ & Before & After & MD & \\
\hline I0-point VAS & $6.3 \pm 0.9$ & $2.7 \pm 1.2$ & $-3.6 \pm 1.4$ & $0.001 * * *$ & $0.02 *$ & $6.3 \pm 0.7$ & $3.2 \pm 0.8$ & $-3.1 \pm 0.8$ & $0.001 * * *$ \\
\hline PPT & $28.5 \pm 5.5$ & $35.7 \pm 6.3$ & $7.2 \pm 5.1$ & $0.001 * * *$ & $0.04 *$ & $27.8 \pm 3.7$ & $32.9 \pm 3.8$ & $5.1 \pm 4.1$ & $0.001 * * *$ \\
\hline ROM (degree) & $33.5 \pm 5.1$ & $35.2 \pm 4.1$ & $1.7 \pm 3.4$ & 0.5 & 0.1 & $32.8 \pm 4.7$ & $33.9 \pm 3.9$ & $1.1 \pm 3.9$ & 0.909 \\
\hline \multirow[t]{3}{*}{ NDI (\%) } & $49.0 \pm 9.5$ & $39.1 \pm 8.5$ & $-9.9 \pm 8.7$ & $0.001 * * *$ & $0.01 * *$ & $46.3 \pm 9.1$ & $40.8 \pm 7.3$ & $-5.5 \pm 3.8$ & $0.001 * * *$ \\
\hline & \multicolumn{3}{|l|}{ LI } & \multirow{2}{*}{\multicolumn{2}{|c|}{$\begin{array}{l}\text { p-value } \\
\text { Before }\end{array}$}} & \multicolumn{3}{|l|}{ OI } & \multirow[t]{2}{*}{$p$-value } \\
\hline & Before & After & MD & & & After & MD & & \\
\hline I0-point VAS & $6.2 \pm 0.9$ & $2.5 \pm I .1$ & $-3.7 \pm 1.5$ & $0.001 * * *$ & & $5.7 \pm 0.9$ & $2.4 \pm 1.5$ & $-3.8 \pm 1.8$ & $0.001 * * *$ \\
\hline PPT & $29.0 \pm 5.2$ & $36.8 \pm 4.6$ & $7.8 \pm 4.3$ & $0.001 * * *$ & & $28.7 \pm 6.9$ & $37.1 \pm 8.5$ & $8.5 \pm 6.1$ & $0.001 * * *$ \\
\hline ROM (degree) & $33.8 \pm 6.5$ & $37.5 \pm 3.0$ & $3.7 \pm 5.2$ & $0.01 * *$ & & $33.9 \pm 4.2$ & $35.9 \pm 3.9$ & $2.0 \pm 5.7$ & 0.104 \\
\hline NDI (\%) & $51.0 \pm 7.0$ & $39.9 \pm 7.9$ & $-11.1 \pm 7.5$ & $0.00 I^{* * * *}$ & & $49.6 \pm 11.4$ & $36.8 \pm 9.8$ & $-|2.8 \pm|||$. & $0.001 * * *$ \\
\hline
\end{tabular}

Note: $* P<0.05, * * P<0.01, * * * P<0.001$.

Abbreviations: ANOVA, analysis of variance; DN, dry needling; LI, lidocaine injection; MD, mean difference; NDI, neck disability index; OI, ozone injection; PPT, pain pressure threshold; ROM, range of motion; VAS, visual analog scale.

$p$-value $=0.001)$, but lateral flexion did not show remarkable increase $(\mathrm{MD}=1.7 \pm 3.4, p$-value $=0.05)$. Cervical $\mathrm{ROM}$ (lateral bending) improvement, except for LI group, which showed a very small MD of 3.7 $\pm 5.2(p$-value $=0.01)$, was not significant.

The maximum improvement was found in VAS $(57 \%$ change), then in PPT (25\%), and finally in NDI (20\%), which were remarkable and clinically important, whereas the least efficacy was seen in cervical lateral flexion ROM (5\%), this was not clinically important. Based on analysis of variance results, there was a statistically significant difference among three groups for VAS, NDI, and PPT ( $p$-value $=0.02,0.01$, and 0.04 , respectively) favoring the ozone and lidocaine groups; the minimum amount of efficacy on all scores (showed as $\mathrm{MD})$ was seen in the $\mathrm{DN}$ group.

In summary, both LI and OI were more effective than DN in improving pain and disability. Although OI group showed slightly higher improvement on VAS, PPT, and NDI compared with LI, no parameters revealed a significant difference between two groups. It should also be mentioned that two participants (one in OI group and another in DN group) had reported some minor adverse events, such as local transient flare reaction, within the first day after injection and without any need for treatment.

\section{Discussion}

To the best of our knowledge, there has not been enough evidence about the use of ozone in MPS patients in the form of a well-designed RCT. However multiple studies have evaluated the efficacy of DN and LI in different settings. In 2014, Rayegani et $\mathrm{a}^{26}$ proved the efficacy of DN treatment accompanied by physiotherapy modalities. In another study, Unalan et $\mathrm{al}^{27}$ aimed to compare the efficacy of LI versus high-power ultrasound; the researchers finally declared that a significant improvement was seen on both pain and ROM within the two groups and that the methods were similar. Similar to this RCT, Hong's study compared the efficacy of DN versus LI in MPS treatment, based on VAS for pain and PPT for tenderness; the authors finally concluded that both were effective (if a local twitch response was elicited) on all parameters, immediately and after 2 weeks. ${ }^{28}$ Another trial by Ay et $\mathrm{al}^{29}$ confirmed almost all these findings at 3 months follow-up as well. In the current survey, we evaluated the efficacy and safety of the three methods after 4 weeks followup; as previously mentioned, we detected an acceptable significant improvement in VAS, PPT, and NDI parameters for all methods, even in DN group, which showed less efficacy compared with the other two treatments. Similarly, a trial in 2010 compared the efficacy of three methods, including LI, $\mathrm{DN}$, and botulinum injection, at 1 month follow-up in MPS patients; the key findings were pain and ROM improvement in all groups and improved fatigue and disability in only LI and botulinum groups. ${ }^{30}$

A systematic review in 2012, done by Ong et al, ${ }^{31}$ that included five RCTs on comparing DN versus LI concluded that although no significant difference was seen between two methods, there were interesting patterns favoring LI immediately after injection and DN at 3-6 months after treatment. However, another meta-analysis in 2013 by Kietrys et $\mathrm{al}^{6}$ yielded slightly different results and reported that LI was more effective than DN on pain improvement immediately and also at 4 weeks follow-up. Despite the incongruent results 
found in existing literatures on the efficacy of DN and LI in treatment of patients with MPS, altogether they have proved that both injections have demonstrated better results compared with the placebo group, ${ }^{6,31}$ with a slightly preference favoring LI, quite similar to our findings.

According to our results, it could be concluded that although all three therapeutic methods significantly improved patients' pain, pressure threshold, and disability at 4 weeks follow-up, cervical lateral flexion range did not show remarkable improvement. Current findings revealed that there was significant difference among the groups regarding their VAS, PPT, and NDI changes; in fact, the DN group showed the least efficacy, but despite a slightly higher improvement in the OI group, no significant difference was found between LI and OI groups.

\section{Limitation}

Despite introducing ozone and comparing its efficacy versus two available treatments, this study had some limitations such as lack of an objective functionality assessment tool and short follow-up period that resulted in inadequate data on evaluating long-term efficacy. Since neither chronic nor acute toxicity has been reported for ozone intramuscular injection, ${ }^{25}$ future studies can assess this new method versus other well-documented treatments or even in combination therapy regimens.

\section{Conclusion}

Based on our findings, three methods, including DN, OI, and LI, were effective in MPS patient treatment at 1-month follow-up; although the ozone group had slightly better results than the LI up to 4 weeks follow-up, the two treatments had no significant difference and both were more successful than DN. Due to the high costs and low accessibility of ozone, further high-quality RCTs with longer follow-up are needed to evaluate whether this injection could be an economical alternative treatment in MPS.

\section{Disclosure}

The authors report no conflicts of interest in this work.

\section{References}

1. Hong CZ. Treatment of myofascial pain syndrome. Curr Pain Headache Rep. 2006;10(5):345-349.

2. Tunks E, McCain GA, Hart LE, et al. The reliability of examination for tenderness in patients with myofascial pain, chronic fibromyalgia and controls. J Rheumatol. 1995;22:944-952.

3. Wolfe F, Simons DG, Fricton J, et al. The fibromyalgia and myofascial pain syndromes: a preliminary study of tender points and trigger points in persons with fibromyalgia, myofascial pain syndrome and no disease. J Rheumatol. 1992;19:944-951.

4. Basford JR, An KN. New techniques for the quantification of fibromyalgia and myofascial pain. Curr Pain Headache Rep. 2009;13:376.
5. Lucas N, Macaskill P, Irwig L, et al. Reliability of physical examination for diagnosis of myofascial trigger points: a systematic review of the literature. Clin J Pain. 2009;25:80-89.

6. Kietrys DM, Palombaro KM, Azzaretto E, et al. Effectiveness of dry needling for upper-quarter myofascial pain: a systematic review and meta-analysis. J Orthop Sports Phys Ther. 2013;43(9):620-634.

7. Shah JP, Thaker N, Heimur J, Aredo JV, Sikdar S, Gerber L . Myofascial trigger points then and now: a historical and scientific perspective. $P M R$. 2015;7(7):746-761.

8. Cho IT, Cho YW, Kwak SG, Chang MC. Comparison between ultrasound-guided interfascial pulsed radiofrequency and ultrasound-guided interfascial block with local anesthetic in myofascial pain syndrome of trapezius muscle. Medicine. 20017;96(5):e6019.

9. Fleckenstein J, Zaps D, Rüger LJ, et al. Discrepancy between prevalence and perceived effectiveness of treatment methods in myofascial pain syndrome: results of a cross-sectional, nationwide survey. $B M C$ Musculoskelet Disord. 2010;11:32.

10. Money S. Pathophysiology of trigger points in Myofascial pain syndrome pathophysiology of trigger points in Myofascial pain syndrome. J Pain Palliat Care Pharmacother. 2017;31(2):158-159.

11. Wheeler AH. Myofascial pain disorders: theory to therapy. Drugs. 2004;64:45-62.

12. Tough EA, White AR, Cummings TM, Richards SH, Campbell JL. Acupuncture and dry needling in the management of myofascial trigger point pain: a systematic review and meta analysis of randomized controlled trials. Eur J Pain. 2009;13:3-10.

13. Peloso P, Gross A, Haines T, Trinh K, Goldsmith CH, Burnie S. Medicinal and injection therapies for mechanical neck disorders. Cochrane Database Syst Rev. 2007;CD000319.

14. Ho KY, Tan KH. Botulinum toxin A for myofascial trigger point injection: a qualitative systematic review. Eur J Pain. 2007;11:519-527.

15. Bellamy N, Buchanan WW, Goldsmith $\mathrm{CH}$, et al. Validation study of WOMAC: a health status instrument for measuring clinically important patient relevant outcomes to antirheumatic drug therapy in patients with osteoarthritis of the hip or knee. J Rheumatol. 1988;15:1833-1840.

16. Srbely JZ, Kumbhare D, Grosman-Rimon L. A narrative review of new trends in the diagnosis of myofascial trigger points: diagnostic ultrasound imaging and biomarkers. J Can Chiropr Assoc. 2016;60(3):220-225.

17. Gerwin RD. Classification, epidemiology, and natural history of myofascial pain syndrome. Curr Pain Headache Rep. 2001;5(5):412-420.

18. Babaei-Ghazani A, Karimi N, Forogh B, et al. Comparison of ultrasoundguided local ozone (O2-O3) injection vs corticosteroid injection in the treatment of chronic plantar fasciitis: a randomized clinical trial. Pain Med. Epub 2018 May 30.

19. Lopes de Jesus CC, dos Santos FC, de Jesus LMOB, Monteiro I, Sant'Ana MSSC, Trevisani VFM. Comparison between intra-articular ozone and placebo in the treatment of knee osteoarthritis: a randomized, doubleblinded, placebo-controlled study. PLoS One. 2017;12(7):e0179185.

20. Raeissadat SA, Rayegani SM, Babaee M, Ghorbani E. The effect of platelet-rich plasma on pain, function, and quality of life of patients with knee osteoarthritis. Pain Res Treat. 2013;2013:165967.

21. Rayegani SM, Raeissadat SA, Taheri MS, et al. Does intra articular platelet rich plasma injection improve function, pain and quality of life in patients with osteoarthritis of the knee? A randomized clinical trial. Orthop Rev (Pavia). 2014;6(3):5405.

22. Richmond J, Hunter D, Irrgang J, et al. American Academy of Orthopaedic Surgeons clinical practice guideline on the treatment of osteoarthritis (OA) of the knee. J Bone Joint Surg Am. 2010;92:990-993.

23. Rodriguez-Merchan EC. Intra-articular injections of hyaluronic acid and other drugs in the knee joint. HSS J. 2013;9(2):180-182.

24. Raeissadat SA, Rayegani SM, Forogh B, Hassan Abadi P, Moridnia M, Rahimi Dehgolan S. Intra-articular ozone or hyaluronic acid injection: which one is superior in patients with knee osteoarthritis? A 6-month randomized clinical trial. J Pain Res. 2018;11:111-117.

25. Borrelli E, Alexandre A, Iliakis E, Alexandre A, Bocci V. Disc Herniation and knee arthritis as chronic oxidative stress diseases: the therapeutic role of oxygen ozone therapy. J Arthritis. 2015;4:161. 
26. Rayegani SM, Bayat M, Bahrami MH, Raeissadat SA, Kargozar E. Comparison of dry needling and physiotherapy in treatment of myofascial pain syndrome. Clin Rheumatol. 2014;33(6):859-864.

27. Unalan H, Majlesi J, Aydin FY, Palamar D. Comparison of high-power pain threshold ultrasound therapy with local injection in the treatment of active myofascial trigger points of the upper trapezius muscle. Arch Phys Med Rehabil. 2011;92(4):657-662.

28. Hong CZ. Lidocaine injection versus dry needling to myofascial trigger point. The importance of the local twitch response. Am J Phys Med Rehabil. 1994;73:256-263.
29. Ay S, Evcik D, Tur BS. Comparison of injection methods in myofascial pain syndrome: a randomized controlled trial. Clin Rheumatol. 2010;29: 19-23.

30. Kamanli A, Kaya A, Ardicoglu O, Ozgocmen S, Zengin FO, Bayik Y. Comparison of lidocaine injection, botulinum toxin injection, and dry needling to trigger points in myofascial pain syndrome. Rheumatol Int. 2005;25:604-611.

31. Ong J, Claydon LS. The effect of dry needling for myofascial trigger points in the neck and shoulders: a systematic review and meta-analysis. J Bodyw Mov Ther. 2014;18(3):390-398.
Journal of Pain Research

\section{Publish your work in this journal}

The Journal of Pain Research is an international, peer reviewed, open access, online journal that welcomes laboratory and clinical findings in the fields of pain research and the prevention and management of pain. Original research, reviews, symposium reports, hypothesis formation and commentaries are all considered for publication

\section{Dovepress}

The manuscript management system is completely online and includes a very quick and fair peer-review system, which is all easy to use. Visit http://www.dovepress.com/testimonials.php to read real quotes from published authors. 